\title{
Conceptualizing and measuring youth-adult partnership in community programs: a cross national study
}

\begin{abstract}
Youth participation in program and community decision making is framed by scholars as an issue of social justice, a platform for positive youth development and effective citizenry, and a strategy for nation building. Recent literature reviews have consistently identified youthadult partnership (Y-AP) as an effective type of youth participation across highly diverse contexts. These same reviews, however, note that indicators of Y-AP have not been conceptualized and validated for measurement purposes. The present study addresses this limitation by developing a brief measure of $\mathrm{Y}-\mathrm{AP}$ that is explicitly grounded in current theory, research, and community practice. The measure was administered to youth in the United States, Malaysia, and Portugal $(\mathrm{N}=610)$. Validation was assessed through factor analysis and tests of factorial, discriminant, and concurrent validity. Results confirmed the two predicted dimensions of the Y-AP measure: youth voice in decision making and supportive adult relationships. These two dimensions were also found to be distinct from other measures of program quality: safety and engagement. As predicted, they also significantly correlated with measures of agency and empowerment. It is concluded that the measure has the potential to support community efforts to maximize the quality of youth programs.
\end{abstract}

Keyword: Measurement validation; Youth participation; Youth voice; Youth-adult partnership 